\title{
Complexity results for generating subgraphs
}

\author{
Vadim E. Levit \\ Department of Computer Science \\ Ariel University, ISRAEL \\ levitv@ariel.ac.il \\ David Tankus \\ Department of Software Engineering \\ Sami Shamoon College of Engineering, ISRAEL \\ davidt@sce.ac.il
}

\begin{abstract}
A graph $G$ is well-covered if all its maximal independent sets are of the same cardinality. Assume that a weight function $w$ is defined on its vertices. Then $G$ is $w$-well-covered if all maximal independent sets are of the same weight. For every graph $G$, the set of weight functions $w$ such that $G$ is $w$-well-covered is a vector space, denoted $W C W(G)$.

Let $B$ be a complete bipartite induced subgraph of $G$ on vertex sets of bipartition $B_{X}$ and $B_{Y}$. Then $B$ is generating if there exists an independent set $S$ such that $S \cup B_{X}$ and $S \cup B_{Y}$ are both maximal independent sets of $G$. In the restricted case that a generating subgraph $B$ is isomorphic to $K_{1,1}$, the unique edge in $B$ is called a relating edge.

Deciding whether an input graph $G$ is well-covered is co-NP-complete. Therefore finding $W C W(G)$ is co-NP-hard. Deciding whether an edge is relating is NP-complete. Therefore, deciding whether a subgraph is generating is NP-complete as well.

In this article we discuss the connections among these problems, provide proofs for NP-completeness for several restricted cases, and present polynomial characterizations for some other cases.
\end{abstract}

Keywords: weighted well-covered graph; maximal independent set; relating edge; generating subgraph; vector space.

\section{Introduction}

\subsection{Basic definitions and notation}

Throughout this paper $G$ is a simple (i.e., a finite, undirected, loopless and without multiple edges) graph with vertex set $V(G)$ and edge set $E(G)$.

Cycles of $k$ vertices are denoted by $C_{k}$. When we say that $G$ does not contain $C_{k}$ for some $k \geq 3$, we mean that $G$ does not admit subgraphs isomorphic to 
$C_{k}$. Note that these subgraphs are not necessarily induced. Let $\mathcal{G}\left(\widehat{C_{i_{1}}}, . ., \widehat{C_{i_{k}}}\right)$ be the family of all graphs which do not contain $C_{i_{1}}, \ldots, C_{i_{k}}$.

Let $u$ and $v$ be two vertices in $G$. The distance between $u$ and $v$, denoted $d(u, v)$, is the length of a shortest path between $u$ and $v$, where the length of a path is the number of its edges. If $S$ is a non-empty set of vertices, then the distance between $u$ and $S$, is defined as $d(u, S)=\min \{d(u, s): s \in S\}$.

For every positive integer $i$, denote

$$
N_{i}(S)=\{x \in V(G): d(x, S)=i\},
$$

and

$$
N_{i}[S]=\{x \in V(G): d(x, S) \leq i\} .
$$

If $S$ contains a single vertex, $v$, then we abbreviate $N_{i}(\{v\}), N_{i}[\{v\}]$ to be $N_{i}(v), N_{i}[v]$, respectively. We denote by $G[S]$ the subgraph of $G$ induced by $S$. For every two sets, $S$ and $T$, of vertices of $G$, we say that $S$ dominates $T$ if $T \subseteq N_{1}[S]$.

\subsection{Well-covered graphs}

Let $G$ be a graph. A set of vertices $S$ is independent if its elements are pairwise nonadjacent. An independent set of vertices is maximal if it is not a subset of another independent set. An independent set of vertices is maximum if the graph does not contain an independent set of a higher cardinality.

The graph $G$ is well-covered if every maximal independent set is maximum [12. Assume that a weight function $w: V(G) \longrightarrow \mathbb{R}$ is defined on the vertices of $G$. For every set $S \subseteq V(G)$, define

$$
w(S)=\sum_{s \in S} w(s) .
$$

Then $G$ is $w$-well-covered if all maximal independent sets of $G$ are of the same weight.

The problem of finding a maximum independent set is NP-complete. However, if the input is restricted to well-covered graphs, then a maximum independent set can be found in polynomial time using the greedy algorithm. Similarly, if a weight function $w: V(G) \longrightarrow \mathbb{R}$ is defined on the vertices of $G$, and $G$ is $w$-well-covered, then finding a maximum weight independent set is a polynomial problem. There is an interesting application, where well-covered graphs are investigated in the context of distributed $k$-mutual exclusion algorithms 18 .

The recognition of well-covered graphs is known to be co-NP-complete. This is proved independently in [5] and [15]. In [4] it is proven that the problem remains co-NP-complete even when the input is restricted to $K_{1,4}$-free graphs. However, the problem can be solved in polynomial time for $K_{1,3}$-free graphs [16, 17, for graphs with girth 5 at least [6], for graphs with a bounded maximal degree [3], for chordal graphs [13], and for graphs without cycles of lengths 4 and 5 [7. 
For every graph $G$, the set of weight functions $w$ for which $G$ is $w$-wellcovered is a vector space [3]. That vector space is denoted $W C W(G)[2$. Since recognizing well-covered graphs is co-NP-complete, finding the vector space $W C W(G)$ of an input graph $G$ is co-NP-hard. However, finding $W C W(G)$ can be done in polynomial time when the input is restricted to graphs with a bounded maximal degree [3], to graphs without cycles of lengths 4,5 and 6 [1], and to chordal graphs [1].

\subsection{Generating subgraphs and relating edges}

Further we make use of the following notions, which have been introduced in 8. Let $B$ be an induced complete bipartite subgraph of $G$ on vertex sets of bipartition $B_{X}$ and $B_{Y}$. Assume that there exists an independent set $S$ such that each of $S \cup B_{X}$ and $S \cup B_{Y}$ is a maximal independent set of $G$. Then $B$ is a generating subgraph of $G$, and the set $S$ is a witness that $B$ is generating. We observe that every weight function $w$ such that $G$ is $w$-well-covered must satisfy the restriction $w\left(B_{X}\right)=w\left(B_{Y}\right)$.

If the generating subgraph $B$ contains only one edge, say $x y$, it is called a relating edge. In such a case, the equality $w(x)=w(y)$ is valid for every weight function $w$ such that $G$ is $w$-well-covered.

Recognizing relating edges is known to be NP-complete 2, and it remains NP-complete even when the input is restricted to graphs without cycles of lengths 4 and 5 [9]. Therefore, recognizing generating subgraphs is also NPcomplete when the input is restricted to graphs without cycles of lengths 4 and 5. However, recognizing relating edges can be done in polynomial time if the input is restricted to graphs without cycles of lengths 4 and $6[9$, and to graphs without cycles of lengths 5 and 6 [11].

It is also known that recognizing generating subgraphs is a polynomial problem when the input is restricted to graphs without cycles of lengths 4, 6 and 7 [8], to graphs without cycles of lengths 4,5 and 6 [11, and to graphs without cycles of lengths 5,6 and 7 [11].

\subsection{Introducing the problems under consideration}

The subject of this article is the following four problems and their interconnections.

- WC problem:

Input: A graph $G$.

Question: Is $G$ well-covered?

- WCW problem:

Input: A graph $G$.

Output: The vector space $W C W(G)$.

- GS problem:

Input: A graph $G$, and an induced complete bipartite subgraph $B$ of $G$. 
Question: Is $B$ generating?

- RE problem:

Input: A graph $G$, and an edge $x y \in E(G)$.

Question: Is $x y$ relating?

If we know the output of the WCW problem for a graph $G$, then we know the output of the WC problem for the same $G$ : The graph $G$ is well-covered if and only if $w \equiv 1$ belongs to $W C W(G)$. Therefore, the WC problem is not harder than the WCW problem. Let $\Psi$ be a family of graphs. If the WCW problem can be solved in polynomial time, when its input is restricted to $\Psi$, then also the $\mathbf{W C}$ problem is polynomial, when its input is restricted to $\Psi$. On the other hand, if the WC problem is co-NP-complete, when its input is restricted to $\Psi$, then the WCW problem is co-NP-hard, when its input is restricted to $\Psi$.

A similar connection exists between the GS problem and the $\mathbf{R E}$ problem, since the $\mathbf{R E}$ problem is a restricted case of the GS problem. Therefore, for every family $\Psi$ of graphs, if the GS problem can be solved in polynomial time, then the $\mathbf{R E}$ problem can be solved in polynomial time as well, and if the $\mathbf{R E}$ problem is NP-complete then the GS problem is also NP-complete.

This article considers bipartite graphs, graphs with girth 6 at least, and $K_{1,4^{-}}$ free graphs. Although for bipartite graphs and graphs with girth 6 at least, the WC problem is known to be solvable in polynomial time, we prove that the GS problem is NP-complete. For bipartite graphs, even the RE problem is NP-complete. Additionally, NP-completeness of the GS problem for $K_{1,4}$-free graphs is proved. We also present polynomial algorithms for the $\mathbf{R E}$ problem, the GS problem, and the WCW problem in the case that the maximum degree of the input graph is bounded.

\section{NP-complete cases}

A binary variable is a variable whose value is either 0 or 1 . If $x$ is a binary variable, then its negation is denoted by $\bar{x}$. Each of $x$ and $\bar{x}$ are called literals. Let $X=\left\{x_{1}, \ldots, x_{n}\right\}$ be a set of binary variables. A clause $c$ over $X$ is a set of literals belonging to $\left\{x_{1}, \overline{x_{1}}, \ldots, x_{n}, \overline{x_{n}}\right\}$ such that $c$ does not contain both a variable and its negation. A truth assignment is a function

$$
\Phi:\left\{x_{1}, \overline{x_{1}}, \ldots, x_{n}, \overline{x_{n}}\right\} \longrightarrow\{0,1\}
$$

such that

$$
\Phi\left(\overline{x_{i}}\right)=1-\Phi\left(x_{i}\right) \text { for each } 1 \leq i \leq n .
$$

A truth assignment $\Phi$ satisfies a clause $c$ if $c$ contains at least one literal $l$ such that $\Phi(l)=1$. 


\subsection{Relating edges in bipartite graphs}

In this subsection we consider the following problems:

\section{- SAT problem:}

Input: A set $X$ of binary variables and a set $C$ of clauses over $X$.

Question: Is there a truth assignment for $X$ which satisfies all clauses of $C ?$

\section{- BWSAT problem:}

Input: $\mathrm{A}$ set $X$ of binary variables and two sets, $C_{1}$ and $C_{2}$, of clauses over $X$, such that all literals of the clauses belonging to $C_{1}$ are variables, and all literals of clauses belonging to $C_{2}$ are negations of variables.

Question: Is there a truth assignment for $X$, which satisfies all clauses of $C_{1} \cup C_{2}$ ?

By Cook-Levin's Theorem, the SAT problem is NP-complete. We prove that the same holds for the BWSAT problem.

Lemma 2.1 The $\boldsymbol{B} \boldsymbol{W S A T}$ problem is in $\boldsymbol{N P}$-complete.

Proof. Obviously, the BWSAT problem is in NP. We prove its NP-completeness by showing a reduction from the $\mathbf{S A T}$ problem. Let

$$
I_{1}=\left(X=\left\{x_{1}, \ldots, x_{n}\right\}, C=\left\{c_{1}, \ldots, c_{m}\right\}\right)
$$

be an instance of the SAT problem. Define $Y=\left\{x_{1}, \ldots, x_{n}, y_{1}, \ldots, y_{n}\right\}$, where $y_{1}, \ldots, y_{n}$ are new variables. For every $1 \leq j \leq m$, let $c_{j}^{\prime}$ be the clause obtained from $c_{j}$ by replacing $\overline{x_{i}}$ with $y_{i}$ for each $1 \leq i \leq n$. Let $C^{\prime}=\left\{c_{1}^{\prime}, \ldots, c_{m}^{\prime}\right\}$. For each $1 \leq i \leq n$ define two new clauses, $d_{i}=\left\{x_{i}, y_{i}\right\}$ and $e_{i}=\left\{\overline{x_{i}}, \overline{y_{i}}\right\}$. Let $D=$ $\left\{d_{1}, \ldots, d_{n}\right\}$ and $E=\left\{e_{1}, \ldots, e_{n}\right\}$. Obviously, all literals of $C^{\prime} \cup D$ are variables, and all literals of $E$ are negations of variables. Hence, $I_{2}=\left(Y, C^{\prime} \cup D, E\right)$ is an instance of the BWSAT problem, see Example 2.2. It remains to prove that $I_{1}$ and $I_{2}$ are equivalent.

Assume that $I_{1}$ is a positive instance of the SAT problem. There exists a truth assignment

$$
\Phi_{1}:\left\{x_{1}, \overline{x_{1}}, \ldots, x_{n}, \overline{x_{n}}\right\} \longrightarrow\{0,1\}
$$

which satisfies all clauses of $C$. Extend $\Phi_{1}$ to a truth assignment

$$
\Phi_{2}:\left\{x_{1}, \overline{x_{1}}, \ldots, x_{n}, \overline{x_{n}}, y_{1}, \overline{y_{1}}, \ldots, y_{n}, \overline{y_{n}}\right\} \longrightarrow\{0,1\}
$$

by defining $\Phi_{2}\left(y_{i}\right)=1-\Phi_{1}\left(x_{i}\right)$ for each $1 \leq i \leq n$. Clearly, $\Phi_{2}$ is a truth assignment which satisfies all clauses of $C^{\prime} \cup D \cup E$. Hence, $I_{2}$ is a positive instance of the BWSAT problem.

Assume $I_{2}$ is a positive instance of the BWSAT problem. There exists a truth assignment

$$
\Phi_{2}:\left\{x_{1}, \overline{x_{1}}, \ldots, x_{n}, \overline{x_{n}}, y_{1}, \overline{y_{1}}, \ldots, y_{n}, \overline{y_{n}}\right\} \longrightarrow\{0,1\}
$$


that satisfies all clauses of $C^{\prime} \cup D \cup E$. For every $1 \leq i \leq n$ it holds that $\Phi_{2}\left(y_{i}\right)=1-\Phi_{2}\left(x_{i}\right)$, or otherwise one of $d_{i}$ and $e_{i}$ is not satisfied. Therefore, $I_{1}$ is a positive instance of the $\mathbf{S A T}$ problem.

Example 2.2 The following contains both an instance of the $\boldsymbol{S A T}$ problem and an equivalent instance of the $\boldsymbol{B} \boldsymbol{W} \boldsymbol{S A T}$ problem.

$I_{1}=(X, C)$, where $X=\left\{x_{1}, x_{2}, x_{3}, x_{4}, x_{5}\right\}$,

$C=\left\{\left\{x_{1}, \overline{x_{2}}, x_{3}\right\},\left\{x_{1}, x_{3}, x_{4}, x_{5}\right\},\left\{\overline{x_{1}}, x_{2}, \overline{x_{3}}, x_{4}\right\},\left\{x_{1}, x_{2}, \overline{x_{4}}, \overline{x_{5}}\right\}\right\}$,

$I_{2}=\left(Y, C_{1}, C_{2}\right)$, where $Y=\left\{x_{1}, x_{2}, x_{3}, x_{4}, x_{5}, y_{1}, y_{2}, y_{3}, y_{4}, y_{5}\right\}$,

$C_{1}=\left\{\left\{x_{1}, y_{2}, x_{3}\right\},\left\{x_{1}, x_{3}, x_{4}, x_{5}\right\},\left\{y_{1}, x_{2}, y_{3}, x_{4}\right\},\left\{x_{1}, x_{2}, y_{4}, y_{5}\right\},\left\{x_{1}, y_{1}\right\}\right.$,

$\left.\left\{x_{2}, y_{2}\right\},\left\{x_{3}, y_{3}\right\},\left\{x_{4}, y_{4}\right\},\left\{x_{5}, y_{5}\right\}\right\}$.

$C_{2}=\left\{\left\{\overline{x_{1}}, \overline{y_{1}}\right\},\left\{\overline{x_{2}}, \overline{y_{2}}\right\},\left\{\overline{x_{3}}, \overline{y_{3}}\right\},\left\{\overline{x_{4}}, \overline{y_{4}}\right\},\left\{\overline{x_{5}}, \overline{y_{5}}\right\}\right\}$

The following theorem is the main result of this section.

Theorem 2.3 The $\mathbf{R E}$ problem is $\mathbf{N P}$-complete even if its input is restricted to bipartite graphs.

Proof. The problem is obviously in NP. We prove NP-completeness by showing a reduction from the BWSAT problem. Let

$$
I_{1}=\left(X=\left\{x_{1}, \ldots, x_{n}\right\}, C_{1}, C_{2}\right)
$$

be an instance of the BWSAT problem, where $C_{1}=\left\{c_{1}, \ldots, c_{m}\right\}$ is a set of clauses which contain only variables, and $C_{2}=\left\{c_{1}^{\prime}, \ldots, c_{m^{\prime}}^{\prime}\right\}$ is a set of clauses which contain only negations of variables. Define a graph $B$ as follows:

$$
\begin{gathered}
V(B)=\{x, y\} \cup\left\{v_{j}: 1 \leq j \leq m\right\} \cup\left\{v_{j}^{\prime}: 1 \leq j \leq m^{\prime}\right\} \cup \\
\left\{u_{i}: 1 \leq i \leq n\right\} \cup\left\{u_{i}^{\prime}: 1 \leq i \leq n\right\}, \\
E(B)=\{x y\} \cup\left\{x v_{j}: 1 \leq j \leq m\right\} \cup\left\{y v_{j}^{\prime}: 1 \leq j \leq m^{\prime}\right\} \cup \\
\left\{v_{j} u_{i}: x_{i} \text { appears in } c_{j}\right\} \cup\left\{v_{j}^{\prime} u_{i}^{\prime}: \overline{x_{i}} \text { appears in } c_{j}^{\prime}\right\} \cup\left\{u_{i} u_{i}^{\prime}: 1 \leq i \leq n\right\} .
\end{gathered}
$$

Clearly, $B$ is bipartite, and the vertex sets of its bipartition are

$$
\left\{u_{i}: 1 \leq i \leq n\right\} \cup\{x\} \cup\left\{v_{j}^{\prime}: 1 \leq j \leq m^{\prime}\right\}
$$

and

$$
\left\{v_{j}: 1 \leq j \leq m\right\} \cup\{y\} \cup\left\{u_{i}^{\prime}: 1 \leq i \leq n\right\} .
$$

Consider the instance $I_{2}=(B, x y)$ of the $\mathbf{R E}$ problem. It is necessary to prove that $I_{1}$ and $I_{2}$ are equivalent.

Assume that $I_{1}$ is a positive instance of the BWSAT problem. Let

$$
\Phi:\left\{x_{1}, \overline{x_{1}}, \ldots, x_{n}, \overline{x_{n}}\right\} \longrightarrow\{0,1\}
$$


be a truth assignment which satisfies all clauses of $C_{1} \cup C_{2}$. Let

$$
S=\left\{u_{i}: \Phi\left(x_{i}\right)=1\right\} \cup\left\{u_{i}^{\prime}: \Phi\left(x_{i}\right)=0\right\} .
$$

Obviously, $S$ is independent. Since $\Phi$ satisfies all clauses of $C_{1} \cup C_{2}$, every vertex of

$$
\left\{v_{j}: 1 \leq j \leq m\right\} \cup\left\{v_{j}^{\prime}: 1 \leq j \leq m^{\prime}\right\}
$$

is adjacent to a vertex of $S$. Hence, $S \cup\{x\}$ and $S \cup\{y\}$ are maximal independent sets. Therefore, $S$ is a witness that $x y$ is a relating edge, and $I_{2}$ is a positive instance of the RE problem.

On the other hand, assume that $I_{2}$ is a positive instance of the RE problem. Let $S$ be a witness of $x y$. Since $S$ is a maximal independent set of

$$
\left\{u_{i}: 1 \leq i \leq n\right\} \cup\left\{u_{i}^{\prime}: 1 \leq i \leq n\right\},
$$

exactly one of $u_{i}$ and $u_{i}^{\prime}$ belongs to $S$, for every $1 \leq i \leq n$. Let

$$
\Phi:\left\{x_{1}, \overline{x_{1}}, \ldots, x_{n}, \overline{x_{n}}\right\} \longrightarrow\{0,1\}
$$

be a truth assignment defined by: $\Phi\left(x_{i}\right)=1 \Longleftrightarrow u_{i} \in S$. The fact that $S$ dominates

$$
\left\{v_{j}: 1 \leq j \leq m\right\} \cup\left\{v_{j}^{\prime}: 1 \leq j \leq m^{\prime}\right\}
$$

implies that all clauses of $C_{1} \cup C_{2}$ are satisfied by $\Phi$. Therefore, $I_{1}$ is a positive instance of the BWSAT problem.

Example 2.4 Let $I_{1}=\left(X, C_{1}, C_{2}\right)$ be an instance of the $\boldsymbol{B} \boldsymbol{W} \boldsymbol{S A T}$ problem, where $X=\left\{x_{1}, x_{2}, x_{3}, x_{4}, x_{5}, x_{6}\right\}$,

$C_{1}=\left\{\left\{x_{1}, x_{2}, x_{3}\right\},\left\{x_{2}, x_{4}\right\},\left\{x_{1}, x_{4}\right\},\left\{x_{1}, x_{5}, x_{6}\right\},\left\{x_{3}, x_{5}, x_{6}\right\}\right\}$, and

$C_{2}=\left\{\left\{\overline{x_{1}}, \overline{x_{2}}, \overline{x_{3}}\right\},\left\{\overline{x_{2}}, \overline{x_{3}}, \overline{x_{4}}, \overline{x_{5}}\right\},\left\{\overline{x_{2}}, \overline{x_{4}}, \overline{x_{5}}, \overline{x_{6}}\right\}\right\}$

Then $I_{2}=(G, x y)$ is an equivalent instance of the $\mathbf{R E}$ problem, where $G$ is the graph shown in Figure 1. The instance $I_{1}$ is positive because of the satisfying assignment $\Phi$ defined by $\Phi\left(x_{i}\right)=0$ if $i \in\{2,6\}$, and $\Phi\left(x_{i}\right)=1$ otherwise. The corresponding witness that $I_{2}$ is positive is the set $\left\{u_{1}, u_{2}^{\prime}, u_{3}, u_{4}, u_{5}, u_{6}^{\prime}\right\}$.

Corollary 2.5 The GS problem is NP-complete when its input is restricted to bipartite graphs.

\subsection{Graphs with girth 6 at least}

In this subsection we consider the following problems:

\section{- 3-SAT problem:}

Input: A set $X$ of binary variables and a set $C$ of clauses over $X$ such that every clause contains exactly 3 literals.

Question: Is there a truth assignment for $X$ satisfying all clauses of $C$ ? 


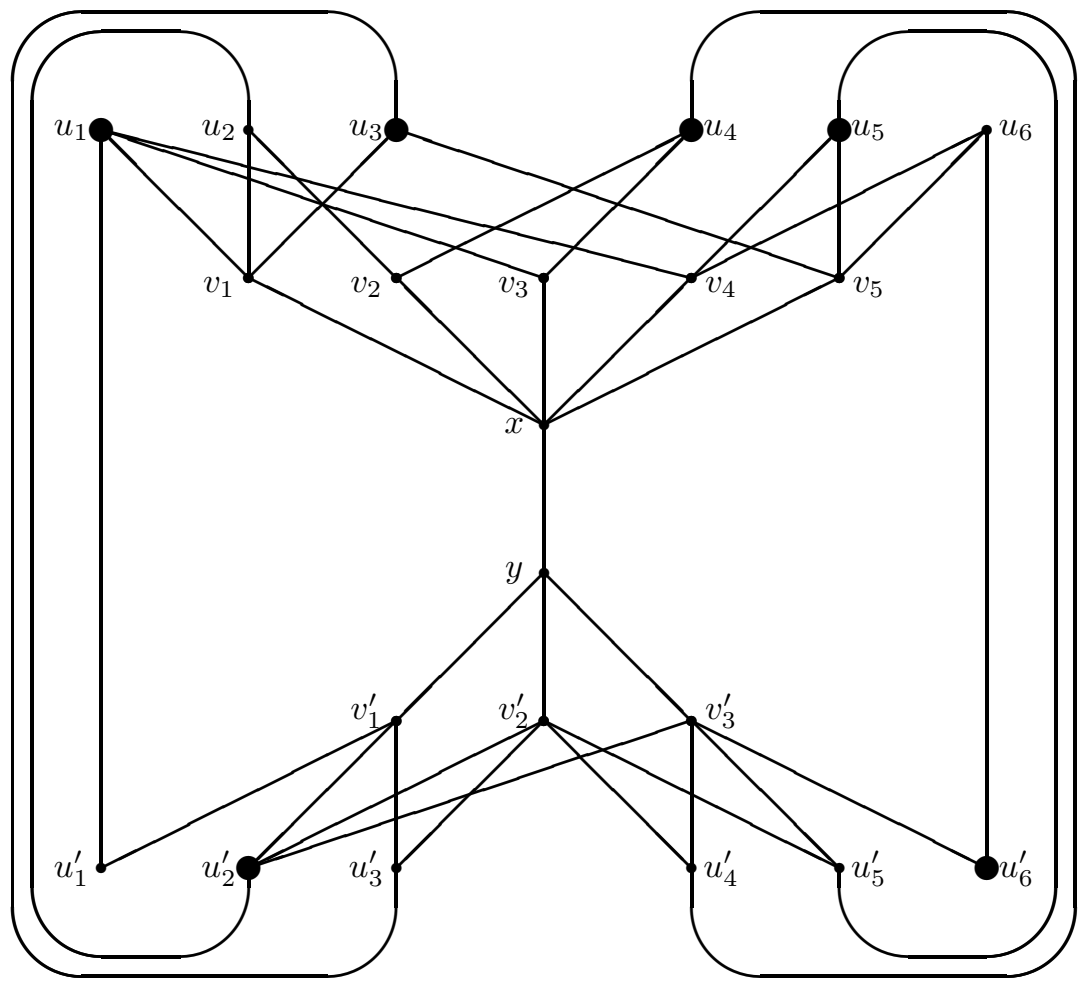

Figure 1: An example of the reduction from the USAT problem to the RE problem.

\section{- DSAT problem:}

Input: A set $X$ of binary variables and a set $C$ of clauses over $X$ such that the following holds:

- Every clause contains 2 or 3 literals.

- Every two distinct clauses have at most one literal in common.

- If two clauses, $c_{1}$ and $c_{2}$, have a common literal $l_{1}$, then there does not exist a literal $l_{2}$ such that $c_{1}$ contains $l_{2}$ and $c_{2}$ contains $\overline{l_{2}}$.

Question: Is there a truth assignment for $X$ satisfying all clauses of $C$ ?

Let $I=(X, C)$ be an instance of the 3-SAT problem. A bad pair of clauses is a set of two clauses $\left\{c_{1}, c_{2}\right\} \subseteq C$ such that there exist literals, $l_{1}, l_{2}, l_{3}, l_{4}, l_{5}$, and:

- $c_{1}=\left\{l_{1}, l_{2}, l_{3}\right\}$ and $c_{2}=\left\{l_{1}, l_{4}, l_{5}\right\}$;

- either $l_{2}=l_{4}$ or $l_{2}=\overline{l_{4}}$. 
Clearly, an instance of the 3-SAT problem with no bad pair of clauses is also an instance of the DSAT problem. The $\mathbf{3 - S A T}$ problem is known to be in NP-complete. We prove that the same holds for the DSAT problem.

Lemma 2.6 The DSAT problem is $\mathbf{N P}$-complete.

Proof. Obviously, the DSAT problem is in NP. We prove its NP-completeness by showing a reduction from the $\mathbf{3 - S A T}$ problem. Let

$$
I_{1}=\left(X=\left\{x_{1}, \ldots, x_{n}\right\}, C=\left\{c_{1}, \ldots, c_{m}\right\}\right)
$$

be an instance of the $\mathbf{3 - S A T}$ problem.

Assume that there exists a bad pair of clauses, $\left\{c_{j_{1}}, c_{j_{2}}\right\} \subseteq C$, i.e. there exist literals, $l_{1}, l_{2}, l_{3}, l_{4}, l_{5}$, such that:

- $c_{j_{1}}=\left\{l_{1}, l_{2}, l_{3}\right\}$ and $c_{j_{2}}=\left\{l_{1}, l_{4}, l_{5}\right\}$;

- either $l_{2}=l_{4}$ or $l_{2}=\overline{l_{4}}$.

Define a new binary variable $x_{n+1}$, and new clauses $c_{j_{2}}^{1}=\left\{l_{1}, x_{n+1}, l_{5}\right\}$, $c_{j_{2}}^{2}=\left\{\overline{l_{4}}, x_{n+1}\right\}$, and $c_{j_{2}}^{3}=\left\{l_{4}, \overline{x_{n+1}}\right\}$. Then

$$
I_{1}^{\prime}=\left(X \cup\left\{x_{n+1}\right\},\left(C \backslash\left\{c_{j_{2}}\right\}\right) \cup\left\{c_{j_{2}}^{1}, c_{j_{2}}^{2}, c_{j_{2}}^{3}\right\}\right)
$$

is an instance of the SAT problem.

We prove that $I_{1}, I_{1}^{\prime}$ are equivalent. Assume that $I_{1}$ is a positive instance of the 3-SAT problem. There exists a truth assignment

$$
\Phi_{1}:\left\{x_{1}, \overline{x_{1}}, \ldots, x_{n}, \overline{x_{n}}\right\} \longrightarrow\{0,1\}
$$

which satisfies all clauses of $C$. Extend $\Phi_{1}$ to a truth assignment

$$
\Phi_{2}:\left\{x_{1}, \overline{x_{1}}, \ldots, x_{n+1}, \overline{x_{n+1}}\right\} \longrightarrow\{0,1\}
$$

by defining $\Phi_{2}\left(x_{n+1}\right)=\Phi_{1}\left(l_{4}\right)$. Clearly, $\Phi_{2}$ satisfies all clauses of $I_{1}^{\prime}$. On the other hand, assume that there exists a truth assignment

$$
\Phi_{2}:\left\{x_{1}, \overline{x_{1}}, \ldots, x_{n+1}, \overline{x_{n+1}}\right\} \longrightarrow\{0,1\}
$$

which satisfies all clauses of $I_{1}^{\prime}$. Clauses $c_{j_{2}}^{2}$, and $c_{j_{2}}^{3}$ imply that $\Phi_{2}\left(x_{n+1}\right)=$ $\Phi_{2}\left(l_{4}\right)$. Therefore, $I_{1}$ is a positive instance of the 3-SAT problem.

The new clauses we added contain a new binary variable. Hence, they do not belong to bad pairs of clauses. Moreover, the clause $c_{j_{2}}$ which belongs to a bad pair in $I_{1}$ was omitted in $I_{1}^{\prime}$. Hence, the number of bad pairs of clauses in $I_{1}^{\prime}$ is smaller than the one in $I_{1}$.

Repeat that process until an instance without bad pairs of clauses is obtained, and denote that instance $I_{2}$. Clearly, every clause of $I_{2}$ has 2 or 3 literals. Hence, $I_{2}$ is an instance of the DSAT problem, and $I_{1}$ and $I_{2}$ are equivalent. 
Example 2.7 The following contains an instance of the 3-SAT problem and an equivalent instance of the $\boldsymbol{D S A T}$ problem.

$I_{1}=\left(X_{1}, C_{1}\right)$ where $X_{1}=\left\{x_{1}, x_{2}, x_{3}, x_{4}, x_{5}\right\}$ and $C_{1}=\left\{\left\{x_{1}, \overline{x_{2}}, x_{3}\right\}\right.$, $\left.\left\{x_{1}, x_{3}, x_{4}\right\},\left\{x_{1}, x_{3}, x_{5}\right\},\left\{\overline{x_{3}}, \overline{x_{4}}, x_{5}\right\},\left\{x_{2}, \overline{x_{3}}, \overline{x_{4}}\right\},\left\{\overline{x_{1}}, \overline{x_{4}}, \overline{x_{5}}\right\}\right\}$.

$I_{2}=\left(X_{2}, C_{2}\right)$ where $X_{2}=\left\{x_{1}, x_{2}, x_{3}, x_{4}, x_{5}, z_{3}, y_{3}, y_{4}, y_{5}\right\}$ and

$C_{2}=\left\{\left\{x_{1}, \overline{x_{2}}, x_{3}\right\},\left\{x_{1}, y_{3}, x_{4}\right\},\left\{x_{1}, z_{3}, x_{5}\right\},\left\{\overline{x_{3}}, \overline{x_{4}}, x_{5}\right\},\left\{x_{2}, \overline{x_{3}}, y_{4}\right\}\right.$,

$\left\{\overline{x_{1}}, \overline{x_{4}}, y_{5}\right\},\left\{\overline{x_{3}}, y_{3}\right\},\left\{x_{3}, \overline{y_{3}}\right\},\left\{\overline{x_{3}}, z_{3}\right\},\left\{x_{3}, \overline{z_{3}}\right\},\left\{x_{4}, y_{4}\right\},\left\{\overline{x_{4}}, \overline{y_{4}}\right\},\left\{x_{5}, y_{5}\right\}$, $\left\{\overline{x_{5}}, \overline{y_{5}}\right\}$.

Theorem 2.8 The following problem is NP-complete:

Input: $A$ graph $G \in \mathcal{G}\left(\widehat{C_{3}}, \widehat{C_{4}}, \widehat{C_{5}}\right)$ and an induced complete bipartite subgraph $B$ of $G$.

Question: Is B generating?

Proof. The problem is obviously in NP. We prove its NP-completeness by showing a reduction from the DSAT problem. Let

$$
I=\left(X=\left\{x_{1}, \ldots, x_{n}\right\}, C=\left\{c_{1}, \ldots, c_{m}\right\}\right)
$$

be an instance of the DSAT problem. Define a graph $G$ as follows.

$$
\begin{gathered}
V(G)=\{y\} \cup\left\{a_{j}: 1 \leq j \leq m\right\} \cup\left\{v_{j}: 1 \leq j \leq m\right\} \cup \\
\left\{u_{i}: 1 \leq i \leq n\right\} \cup\left\{u_{i}^{\prime}: 1 \leq i \leq n\right\} . \\
E(G)=\left\{y a_{j}: 1 \leq j \leq m\right\} \cup\left\{a_{j} v_{j}: 1 \leq j \leq m\right\} \cup\left\{v_{j} u_{i}: x_{i} \text { appears in } c_{j}\right\} \cup \\
\left\{v_{j} u_{i}^{\prime}: \overline{x_{i}} \text { appears in } c_{j}\right\} \cup\left\{u_{i} u_{i}^{\prime}: 1 \leq i \leq n\right\} .
\end{gathered}
$$

Since a clause can not contain both a variable and its negation, $G$ does not contain $C_{3}$. The fact that there are no pairs of bad clauses implies that $G$ does not contain $C_{4}$ and $C_{5}$. Hence, $G \in \mathcal{G}\left(\widehat{C_{3}}, \widehat{C_{4}}, \widehat{C_{5}}\right)$. Let $B=G\left[\{y\} \cup\left\{a_{j}: 1 \leq j \leq m\right\}\right]$. Obviously, $B$ is complete bipartite. Then $J=(G, B)$ is an instance of the GS problem. It remains to prove that $I$ and $J$ are equivalent.

Assume that $I$ is positive, and let

$$
\Phi:\left\{x_{1}, \overline{x_{1}}, \ldots, x_{n}, \overline{x_{n}}\right\} \longrightarrow\{0,1\}
$$

be a truth assignment which satisfies all clauses of $C$. Define

$$
S=\left\{u_{i}: \Phi\left(x_{i}\right)=1\right\} \cup\left\{u_{i}^{\prime}: \Phi\left(x_{i}\right)=0\right\} .
$$

Obviously, $S$ is independent. Since $\Phi$ satisfies all clauses of $C$, the set $S$ dominates $\left\{v_{j}: 1 \leq j \leq m\right\}$. Hence, $S$ is a witness that $B$ is generating, i.e., $J$ is positive.

Assume that $J$ is positive. Let $S$ be a witness that $B$ is generating, and let $S^{*}$ be a maximal independent set of $\left\{u_{i}: 1 \leq i \leq n\right\} \cup\left\{u_{i}^{\prime}: 1 \leq i \leq n\right\}$ which contains $S$. For every $1 \leq i \leq n$, it holds that $\left|S^{*} \cap\left\{u_{i}, u_{i}^{\prime}\right\}\right|=1$. Define

$$
\Phi:\left\{x_{1}, \overline{x_{1}}, \ldots, x_{n}, \overline{x_{n}}\right\} \longrightarrow\{0,1\}
$$


by $\Phi\left(x_{i}\right)=1 \Longleftrightarrow u_{i} \in S^{*}$ for every $1 \leq i \leq n$. Since $S^{*}$ dominates $\left\{v_{j}: 1 \leq j \leq m\right\}$, the function $\Phi$ satisfies all clauses of $C$, and $I$ is a positive instance.

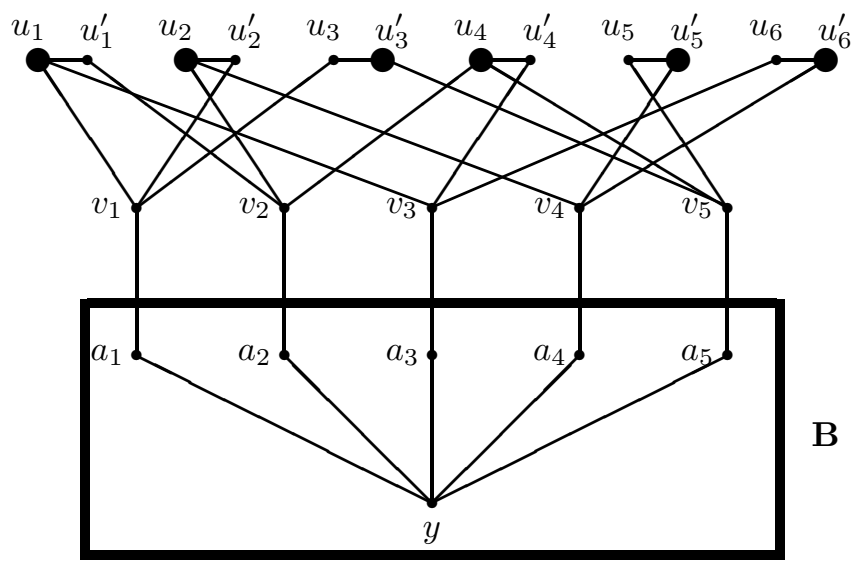

Figure 2: An example of the reduction from the DSAT problem to the GS problem.

Example 2.9 Let $I_{1}=(X, C)$ be an instance of the $\boldsymbol{D S A T}$ problem, where $X=\left\{x_{1}, x_{2}, x_{3}, x_{4}, x_{5}, x_{6}\right\}$ and $C=\left\{\left\{x_{1}, \overline{x_{2}}, x_{3}\right\},\left\{\overline{x_{1}}, x_{2}, x_{4}\right\},\left\{x_{1}, \overline{x_{4}}, x_{6}\right\}\right.$,

$\left.\left\{x_{2}, \overline{x_{5}}, \overline{x_{6}}\right\},\left\{\overline{x_{3}}, x_{4}, x_{5}\right\}\right\}$. Then $I_{2}=(G, B)$ is an equivalent instance of the GS problem, where $G$ and $B$ are the graphs shown in Figure 2. The instance $I_{1}$ is positive because of the satisfying assignment $\Phi$ defined by $\Phi\left(x_{i}\right)=1$ if $i \in\{1,2,4\}$, and $\Phi\left(x_{i}\right)=0$ otherwise. The corresponding witness that $I_{2}$ is positive is the set $\left\{u_{1}, u_{2}, u_{3}^{\prime}, u_{4}, u_{5}^{\prime}, u_{6}^{\prime}\right\}$.

\section{$2.3 \quad K_{1,4}$-free graphs}

Theorem 2.10 [4] The following problem is co-NP-complete:

Input: $A K_{1,4}$-free graph $G$.

Question: Is $G$ well-covered?

We use Theorem 2.10 to prove the following.

Theorem 2.11 The GS problem is $\mathbf{N P}$-complete even when its input is restricted to $K_{1,4}$-free graphs.

Proof. Let $G$ be a $K_{1,4}$-free graph. An induced complete bipartite subgraph of $G$ is isomorphic to $K_{i, j}$, for $1 \leq i \leq j \leq 3$. Hence, the number of these subgraphs is $O\left(n^{6}\right)$, which is polynomial. Every unbalanced induced complete bipartite subgraph of $G$ is a copy of $K_{1,2}$ or $K_{1,3}$ or $K_{2,3}$. The number of these subgraphs is $O\left(n^{5}\right)$. 
Assume, on the contrary, that there exists a polynomial algorithm solving the GS problem for $K_{1,4}$-free graphs. The following algorithm decides in polynomial time whether a $K_{1,4}$-free graph $G$ is well-covered. For each induced complete bipartite unbalanced subgraph $B$ of $G$ on vertex sets of bipartition $B_{X}$ and $B_{Y}$, decide in polynomial time whether $B$ is generating. Once an unbalanced generating subgraph is discovered, the algorithm terminates announcing $G$ is not well-covered. If the algorithm checked all induced complete bipartite unbalanced subgraphs of $G$, and none of them is generating, then $G$ is well-covered. Hence, the WC problem can be solved in polynomial time when its input is restricted to $K_{1,4}$-free graphs, but that contradicts Theorem 2.10. Thus the GS problem is NP-complete, when its input is a $K_{1,4}$-free graph.

\section{Polynomial algorithms when $\Delta$ is bounded}

In this section $G$ will be a graph with $n$ vertices and of maximum degree $\Delta$. The main findings of this section are polynomial algorithms for the $\mathbf{R E}$ problem and the GS problem in the restricted case, when $\Delta$ is bounded. Our motivation here is the following.

Theorem 3.1 [3] Let $k \in N$. The following problem is polynomial. Input: A graph $G$ with $\Delta_{G} \leq k \cdot\left(\log _{2} n\right)^{\frac{1}{3}}$, and a function $w: V(G) \longrightarrow \mathbb{R}$. Question: Is $G$ w-well-covered?

We prove that the GS problem is polynomial, when $\Delta$ is bounded using the same technique as in Theorem 3.1 .

Theorem 3.2 Let $k \in N$. The following problem can be solved in $O\left(n^{2+2 k^{3}}\right)$ time.

Input: A graph $G$ such that $\Delta \leq k \cdot\left(\log _{2} n\right)^{\frac{1}{3}}$, and an induced complete bipartite subgraph $B$ of $G$.

Question: Is B generating?

Proof. Let $B$ be an induced complete bipartite subgraph of $G$ on vertex sets of bipartition $B_{X}$ and $B_{Y}$. For every $V \in\{X, Y\}$, let $U \in\{X, Y\}-\{V\}$, and define:

$$
M_{1}\left(B_{V}\right)=N\left(B_{V}\right) \cap N_{2}\left(B_{U}\right), M_{2}\left(B_{V}\right)=N\left(M_{1}\left(B_{V}\right)\right) \cap N_{2}\left(B_{V}\right) .
$$

Then $\left|M_{1}\left(B_{V}\right)\right| \leq k^{2}\left(\log _{2} n\right)^{2 / 3}$ and $\left|M_{2}\left(B_{V}\right)\right| \leq k^{3} \log _{2} n$. Obviously, $B$ is generating if and only if there exists an independent set in $M_{2}\left(B_{X}\right) \cup M_{2}\left(B_{Y}\right)$ that dominates $M_{1}\left(B_{X}\right) \cup M_{1}\left(B_{Y}\right)$.

The following algorithm decides whether $B$ is generating. For each subset $S$ of $M_{2}\left(B_{X}\right) \cup M_{2}\left(B_{Y}\right)$, check whether $S$ is independent and dominates $M_{1}\left(B_{X}\right) \cup M_{1}\left(B_{Y}\right)$. Once an independent set $S \subseteq M_{2}\left(B_{X}\right) \cup M_{2}\left(B_{Y}\right)$ is found such that $M_{1}\left(B_{X}\right) \cup M_{1}\left(B_{Y}\right) \subseteq N[S]$, the algorithm terminates announcing the instance at hand is positive. If all subsets of $M_{2}\left(B_{X}\right) \cup M_{2}\left(B_{Y}\right)$ were checked, 
and none of them is independent and dominates $M_{1}\left(B_{X}\right) \cup M_{1}\left(B_{Y}\right)$, then the algorithm returns a negative answer.

The number of subsets the algorithm checks is

$$
O\left(2^{\left|M_{2}\left(B_{X}\right) \cup M_{2}\left(B_{Y}\right)\right|}\right)=O\left(2^{2 k^{3} \log _{2} n}\right)=O\left(n^{2 k^{3}}\right) .
$$

For each subset $S$, the decision whether $S$ is both independent and dominates $M_{1}\left(B_{X}\right) \cup M_{1}\left(B_{Y}\right)$ can be done in $O\left(n^{2}\right)$. Therefore, the algorithm terminates in $O\left(n^{2+2 k^{3}}\right)$ time, which is polynomial.

We next prove that the $\mathbf{R E}$ problem is polynomial for the less restrictable bound in comparison with its counterpart from Theorem 3.2 .

Theorem 3.3 Let $k \in N$. The following problem can be solved in $O\left(n^{2+2 k^{2}}\right)$ time.

Input: $A$ graph $G$ such that $\Delta \leq k \cdot\left(\log _{2} n\right)^{\frac{1}{2}}$, and an edge $x y \in E$. Question: Is $x y$ relating?

Proof. For every $v \in\{x, y\}$, let $u \in\{x, y\}-\{v\}$. Define: $M_{1}(v)=N(v) \cap N_{2}(u)$, $M_{2}(v)=N\left(M_{1}(v)\right) \cap N_{2}(v)$. Then $\left|M_{1}(v)\right| \leq k \cdot\left(\log _{2} n\right)^{\frac{1}{2}}$ and $\left|M_{2}(v)\right| \leq$ $k^{2} \log _{2} n$. Clearly, $x y$ is relating if and only if there exists an independent set in $M_{2}(x) \cup M_{2}(y)$, which dominates $M_{1}(x) \cup M_{1}(y)$.

The following algorithm decides whether $x y$ is relating. For each subset $S$ of $M_{2}(x) \cup M_{2}(y)$, check whether $S$ is independent and dominates $M_{1}(x) \cup M_{1}(y)$. Once an independent set $S \subseteq M_{2}(x) \cup M_{2}(y)$ is found such that $M_{1}(x) \cup M_{1}(y) \subseteq N[S]$, the algorithm terminates announcing the instance at hand is positive. If all subsets of $M_{2}(x) \cup M_{2}(y)$ were checked, and none of them is both independent and dominates $M_{1}(x) \cup M_{1}(y)$, then the algorithm returns a negative answer.

The number of subsets the algorithm checks is

$$
O\left(2^{\left|M_{2}(x) \cup M_{2}(y)\right|}\right)=O\left(2^{2 k^{2} \log _{2} n}\right)=O\left(n^{2 k^{2}}\right) .
$$

For each subset $S$, the decision whether $S$ is both independent and dominates $M_{1}(x) \cup M_{1}(y)$ can be done in $O\left(n^{2}\right)$ time. Therefore, the algorithm terminates in $O\left(n^{2+2 k^{2}}\right)$ time.

In what follows, our purpose is both to formalize and to give a detailed proof of a claim mentioned in [3].

Theorem 3.4 Let $k \in N$. The following problem can be solved in $O\left(n^{3+2 k^{2}+2 k^{3}}\right)$ time.

Input: $A$ graph $G$ such that $\Delta \leq k \cdot\left(\log _{2} n\right)^{\frac{1}{3}}$.

Output: The vector space $W C W(G)$.

Proof. Let $G$ be a graph such that $\Delta \leq k \cdot\left(\log _{2} n\right)^{\frac{1}{3}}$. For every vertex $v \in V$, let $L_{v}$ be the vector space of all weight functions $w: V(G) \longrightarrow \mathbb{R}$ which satisfy all restrictions of all generating subgraphs which contain the vertex $v$. Clearly, 
$W C W(G)=\bigcap_{v \in V(G)} L_{v}$. Hence, we first present an algorithm for finding $L_{v}$ for every $v \in V$.

Let $v \in V$. Since the diameter of every complete bipartite graph is at most 2 , every complete bipartite subgraph of $G$ which contains $v$ is a subgraph of $N_{2}[v]$. However,

$$
\left|N_{2}(v)\right| \leq \Delta^{2} \leq k^{2}\left(\log _{2} n\right)^{\frac{2}{3}}
$$

and

$$
\left|N_{2}[v]\right| \leq 2\left|N_{2}(v)\right| \leq 2 k^{2}\left(\log _{2} n\right)^{\frac{2}{3}} .
$$

Therefore, the number of induced complete bipartite subgraphs which contain $v$ cannot exceed

$$
2^{2 k^{2}\left(\log _{2} n\right)^{\frac{2}{3}}} \leq n^{2 k^{2}} .
$$

The following algorithm finds $L_{v}$ :

- For each induced complete bipartite subgraph $B=\left(B_{X}, B_{Y}\right)$ of $G$ containing $v$ :

- Decide whether $B$ is generating;

- If $B$ is generating add the restriction $w\left(B_{X}\right)=w\left(B_{Y}\right)$ to the list of equations defining $L_{v}$.

We have proved that the number of induced complete bipartite subgraphs of $G$ containing $v$ cannot exceed $n^{2 k^{2}}$. By Theorem 3.2, deciding for each subgraph whether it is generating can be done in $O\left(n^{2+2 k^{3}}\right)$ time. Therefore, the algorithm for finding $L_{v}$ terminates in $O\left(n^{2+2 k^{2}+2 k^{3}}\right)$ time. In order to find $W C W(G)$, the algorithm for finding $L_{v}$ should be invoked $n$ times. Therefore, finding $W C W(G)$ can be completed in $O\left(n^{3+2 k^{2}+2 k^{3}}\right)$ time.

\section{Conclusions and future work}

The following table presents complexity results concerning the four major problems presented in this paper. The empty table cells correspond to unsolved cases. In particular, we want to find the complexity status of the WCW problem for bipartite graphs and for graphs with girth 6 at least. For these families of graphs the GS problem is NP-complete while the WC problem is polynomial. Hence, either we obtain a family of graphs for which the $\mathbf{W C}$ problem is polynomial while the WCW problem is co-NP-hard, or we obtain a family of graphs for which the GS problem is NP-complete while the WCW problem is polynomial.

In addition, we are interested in finding some polynomial relaxations of the bipartite case, if any. For instance, can recognizing well-covered graphs belonging to $\mathcal{G}\left(\widehat{C_{3}}, \widehat{C_{5}}\right)$ be done polynomially?

Let us emphasize that we do not know whether there exists a family of graphs for which the $\mathbf{R E}$ problem can be solved in polynomial time, but the GS problem is NP-complete. 


\begin{tabular}{|c|c|c|c|c|}
\hline Input & $\overline{\mathrm{WC}}$ & $\overline{\mathbf{W C W}}$ & $\mathbf{R E}$ & GS \\
\hline general & $\begin{array}{c}\text { co-NPC } \\
5,15\end{array}$ & $\begin{array}{c}\mathbf{c o - N P H} \\
5,15\end{array}$ & $\begin{array}{c}\text { NPC } \\
2\end{array}$ & $\begin{array}{c}\mathbf{N P C} \\
2\end{array}$ \\
\hline$K_{1,3}$-free & $\begin{array}{l}\mathbf{P} \\
{[16]} \\
\end{array}$ & $\begin{array}{l}\mathbf{P} \\
10 \\
\end{array}$ & $\begin{array}{l}\mathbf{P} \\
{[17]}\end{array}$ & $\begin{array}{l}\mathbf{P} \\
17]\end{array}$ \\
\hline$K_{1,4}$-free & $\begin{array}{c}\text { co-NPC } \\
{[4}\end{array}$ & $\begin{array}{c}\text { co-NPH } \\
{[4]}\end{array}$ & & $\begin{array}{l}\text { NPC } \\
\text { this paper }\end{array}$ \\
\hline $\mathcal{G}\left(\widehat{C_{4}}, \widehat{C_{5}}\right)$ & $\begin{array}{l}\mathbf{P} \\
7 \\
\end{array}$ & & $\begin{array}{c}\text { NPC } \\
{[9} \\
\end{array}$ & $\begin{array}{c}\text { NPC } \\
{[9]} \\
\end{array}$ \\
\hline $\mathcal{G}\left(\widehat{C_{4}}, \widehat{C_{6}}\right)$ & & & $\begin{array}{l}\mathbf{P} \\
9 \\
\end{array}$ & \\
\hline $\mathcal{G}\left(\widehat{C_{5}}, \widehat{C_{6}}\right)$ & & & $\begin{array}{l}\mathbf{P} \\
{[11]}\end{array}$ & \\
\hline $\mathcal{G}\left(\widehat{C_{5}}, \widehat{C_{6}}, \widehat{C_{7}}\right)$ & & & $\begin{array}{l}\mathbf{P} \\
11]\end{array}$ & $\begin{array}{l}\mathbf{P} \\
11 \\
\end{array}$ \\
\hline $\mathcal{G}\left(\widehat{C_{4}}, \widehat{C_{5}}, \widehat{C_{6}}\right)$ & $\begin{array}{l}\mathbf{P} \\
7 \\
\end{array}$ & $\begin{array}{l}\mathbf{P} \\
11 \\
11\end{array}$ & $\begin{array}{l}\mathbf{P} \\
11 \\
\end{array}$ & $\begin{array}{l}\mathbf{P} \\
11 \\
\end{array}$ \\
\hline $\mathcal{G}\left(\widehat{C_{4}}, \widehat{C_{6}}, \widehat{C_{7}}\right)$ & & & $\begin{array}{l}\mathbf{P} \\
8 \\
\end{array}$ & $\begin{array}{l}\mathbf{P} \\
8\end{array}$ \\
\hline bipartite & $\begin{array}{l}\mathbf{P} \\
14 \\
\end{array}$ & & $\begin{array}{c}\text { NPC } \\
\text { this paper }\end{array}$ & $\begin{array}{c}\text { NPC } \\
\text { this paper }\end{array}$ \\
\hline $\mathcal{G}\left(\widehat{C_{3}}, \widehat{C_{4}}\right)$ & $\begin{array}{l}\mathbf{P} \\
6 \\
\end{array}$ & & & $\begin{array}{c}\text { NPC } \\
\text { this paper }\end{array}$ \\
\hline $\mathcal{G}\left(\widehat{C_{3}}, \widehat{C_{4}}, \widehat{C_{5}}\right)$ & $\begin{array}{l}\mathbf{P} \\
6] \\
\end{array}$ & & & $\begin{array}{c}\text { NPC } \\
\text { this paper }\end{array}$ \\
\hline$\Delta \leq k\left(\log _{2} n\right)^{\frac{1}{3}}$ & $\begin{array}{l}\mathbf{P} \\
3 \\
\end{array}$ & $\begin{array}{l}\mathbf{P} \\
3 \\
\end{array}$ & $\begin{array}{c}\mathbf{P} \\
{[3] \text { and this paper }}\end{array}$ & $\begin{array}{c}\mathbf{P} \\
{[3] \text { and this paper }}\end{array}$ \\
\hline$\Delta \leq k\left(\log _{2} n\right)^{\frac{1}{2}}$ & & & $\begin{array}{c}\mathbf{P} \\
{[3] \text { and this paper }}\end{array}$ & \\
\hline
\end{tabular}

Table 1: Complexity results on the 4 problems. 
Another interesting open question is whether there exists a family of graphs for which the GS problem is polynomial and its corresponding WCW problem is co-NP-hard.

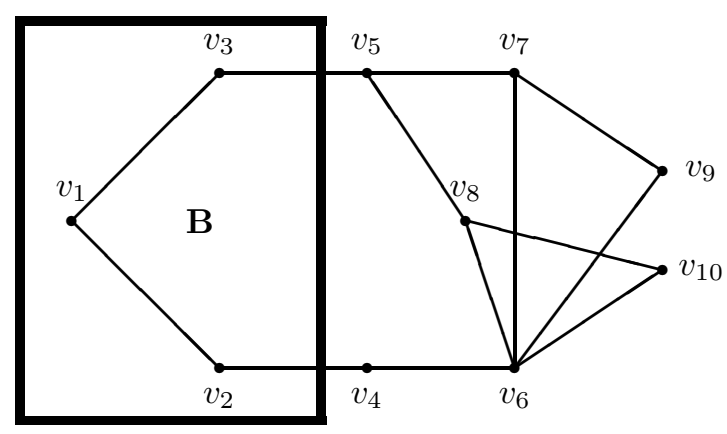

Figure 3: The failure of the naive algorithm.

The naive algorithm for the GS problem, receives as its input an instance $I=\left(G, B=\left(B_{X}, B_{Y}\right)\right)$. Then it finds $W C W(G)$. If there exists a weight function $w \in W C W(G)$ such that $w\left(B_{X}\right) \neq w\left(B_{Y}\right)$, then $B$ is not generating, and consequently, $I$ is negative. Otherwise, $I$ is positive. For every family $\Psi$ of graphs, if the WCW problem can be solved polynomially, then the naive algorithm for the GS problem terminates polynomially.

However, the naive algorithm fails, when its input is $(G, B)$, where $G$ is the graph shown in Figure 3, and $B$ is the subgraph induced by $\left\{v_{1}, v_{2}, v_{3}\right\}$. A function $w: V(G) \longrightarrow \mathbb{R}$ belongs to $W C W(G)$ if and only if the following conditions hold:

- $w\left(v_{7}\right)=w\left(v_{9}\right)$

- $w\left(v_{8}\right)=w\left(v_{10}\right)$

- $w\left(v_{6}\right)=w\left(v_{9}\right)+w\left(v_{10}\right)$

- $w\left(v_{i}\right)=0$ for every $1 \leq i \leq 5$.

Hence, $w\left(v_{1}\right)=w\left(v_{2}\right)+w\left(v_{3}\right)$ for every $w \in W C W(G)$, and the naive algorithm decides that $B$ is generating, although it is not.

\section{References}

[1] J. I. Brown, R. J. Nowakowski, Well covered vector spaces of graphs, SIAM Journal on Discrete Mathematics 19 (2006) 952-965. 
[2] J. I. Brown, R. J. Nowakowski, I. E. Zverovich, The structure of well-covered graphs with no cycles of length 4, Discrete Mathematics 307 (2007) 22352245 .

[3] Y. Caro, N. Ellingham, G. F. Ramey, Local structure when all maximal independent sets have equal weight, SIAM Journal on Discrete Mathematics 11 (1998) 644-654.

[4] Y. Caro, A. Sebő, M. Tarsi, Recognizing greedy structures, Journal of Algorithms 20 (1996) 137-156.

[5] V. Chvatal, P. J. Slater, A note on well-covered graphs, Quo Vadis, Graph Theory?, Annals of Discrete Mathematics 55, North Holland, Amsterdam (1993) 179-182.

[6] A. Finbow, B. Hartnell, R. Nowakowski, A characterization of well-covered graphs of girth 5 or greater, Journal of Combinatorial Theory B 57 (1993) 44-68.

[7] A. Finbow, B. Hartnell, R. Nowakowski, A characterization of well-covered graphs that contain neither 4- nor 5-cycles, Journal of Graph Theory 18 (1994) 713-721.

[8] V. E. Levit, D. Tankus Weighted well-covered graphs without $C_{4}, C_{5}, C_{6}$, $C_{7}$, Discrete Applied Mathematics 159 (2011) 354-359.

[9] V. E. Levit, D. Tankus, On relating edges in graphs without cycles of length 4, Journal of Discrete Algorithms 26 (2014) 28-33.

[10] V. E. Levit, D. Tankus, Weighted well-covered claw-free graphs, Discrete Mathematics 338 (2015) 99-106.

[11] V. E. Levit, D. Tankus, Well-covered graphs without cycles of lengths 4, 5 and 6, Discrete Applied Mathematics 186 (2015) 158-167.

[12] M. D. Plummer, Some covering concepts in graphs, Journal of Combinatorial Theory 8 (1970) 91-98.

[13] E. Prisner, J. Topp and P. D. Vestergaard, Well-covered simplicial, chordal and circular arc graphs, Journal of Graph Theory 21 (1996) 113-119.

[14] G. Ravindra, Well-covered graphs, Journal of Combinatorics, Information and System Sciences 2 (1977) 20-21.

[15] R. S. Sankaranarayana, L. K. Stewart, Complexity results for well-covered graphs, Networks 22 (1992) 247-262.

[16] D. Tankus, M. Tarsi, Well-covered claw-free graphs, Journal of Combinatorial Theory B 66 (1996) 293-302. 
[17] D. Tankus, M. Tarsi, The structure of well-covered graphs and the complexity of their recognition problems, Journal of Combinatorial Theory B 69 (1997) 230-233.

[18] M. Yamashita, T. Kameda, Modeling $k$-coteries by well-covered graphs, Networks 34 (1999) 221-228. 